\title{
Cervical Neoplasia-Cyto-Histological Correlation (Bethesda System) A Study of 276 Cases
}

\author{
Vaishali Jain* and AS Vyas
}

Associate Professor, Department of Pathology, Grant Medical College \& Sir JJ group of hospitals, Byculla, Mumbai, India

\begin{abstract}
Carcinoma uterine cervix is one of the leading causes of cancer death among women worldwide. To detect this widely prevalent cancer at an early stage, the simplest test has been a pap smear. Reporting of pap smears is done by using The Bethesda System 2001 prior to which many classification systems were developed [1,2,3,4]. To check the sensitivity and specificity of Bethesda system, the cytological findings have to be correlated with histology considering histopathology as gold standard. In our study, we have correlated cytological findings (pap smears) with corresponding histology after follow up of the symptomatic patients in the age range of 20 to 80 years. The main objectives were to study usefulness of cytology in detecting various preneoplastic and neoplastic lesions of cervix, to evaluate and to interpret the cases of epithelial lesions according to The Bethesda 2001 classification system [5] and correlation of cytological findings with follow-up histology sections. Analysis of different factors causing discrepancies was done.

Aims: The study was conducted to study usefulness of cervical cytology by PAP smear and the application of the Bethesda 2001 system in classifying different preneoplastic and neoplastic lesions of cervix.

Setting and design: With the use of PAP test and applying the BETHESDA 2001 classification 5 , in the present study we have examined cervical smears of symptomatic patients in the age group ranging from 25 to 70 years. Inflammatory \& unsatisfactory smears were excluded. The remaining cases showing epithelial abnormalities were followed up and biopsy slides were studied. Cytohistological correlation was done in those cases and results were obtained. .
\end{abstract}

Keywords: Cervical cytology; Histology; Correlation

\section{Introduction}

The present study was conducted to study the usefulness of vaginal and cervical cytology in diagnosis of preneoplastic and neoplastic lesions of uterine cervix.

\section{Aims and objectives}

To evaluate and to interpret the cases of epithelial lesions according to The Bethesda 2001 classification system. Correlation of cytological findings with follow-up histology sections. Analysis of different factors causing discrepancies.

The Bethesda system for reporting cervical/vaginal cytological diagnosis is a uniform system for reporting \& it is useful to provide effective communication among cytopathologists \& referring physician. It also facilitates cytological-histopathological correlation. For research into the epidemiology, biology \& pathology of cervical disease, Bethesda system can be applied. From statistical point of view it provides reliable data for national \& international statistical analysis \& comparison.

\section{Subjects and methods}

In this study, the samples for cervical smears were collected from endocervical canal. The smears were made by scrapping the cervix from the squamo-columnar junction with the help of cotton swab stick. The endocervical smears were made by rotating the swab stick in clockwise direction in squamo-columnar junction and stained by PAP stain. The smears showing epithelial abnormality and the follow up biopsy were studied retrospectively. Correlation of cytological findings with histological counterpart was done and concordance rate was calculated for each entity considering histology as the gold standard. The reporting of PAP smears was done according to Bethesda 2001 classification \& for histology WHO classification was used. Comparison was done with other similar studies in the past. The results are presented in tables.

The overall concordance of the study was $70.7 \%$. The discordant cases were $81(29.3 \%)$. The sensitivity was $78 \%$ \& positive predictive value $91.1 \%$ (Table 1 ).
The concordance rate is highest for squamous cell carcinoma which also contributes to increase the overall concordance. For CIN the rate is low $42.2 \%$ (Table 2 ).

\section{Material and Methods}

Present study was carried out on patients attending the Cytology OPD at our hospital over a period of two years i.e. from June 2005 to June 2007. Personal information \& clinical history like age, parity, religion, use of tobacco, socio-economic status, chief complaints, HIV status, any pervious treatment (hormonal /surgery/radiotherapy) taken for carcinoma cervix was noted. The age range of the subjects varied from 20 to 70 yrs. with parity between 0 to $>5$.

Cervical smears were collected from endocervical canal. The

\begin{tabular}{|c|c|c|}
\hline Cases & Number & Percentage \\
\hline Discordant & 81 & 29.3 \\
\hline Concordant & 195 & 70.7 \\
\hline Total & 276 & 100.0 \\
\hline
\end{tabular}

Table 1: The overall concordance rate of the study.

\begin{tabular}{|l|l|}
\hline Concordance & Percentage \\
\hline 1.Overall study & 70.7 \\
\hline 2. Squamous cell carcinoma & 83.6 \\
\hline 3. cervical intraepithelial neoplasia (CIN) & 42.2 \\
\hline
\end{tabular}

Table 2: Comparative concordance rates.

${ }^{*}$ Corresponding author: Dr Vaishali Jain, MD, Associate Professor, Departmen of Pathology, Grant Medical College \& Sir JJ group of hospitals, Byculla, Mumbai India, E-mail: drvaishali123@gmail.com

Received October 07, 2010; Accepted November 23, 2010; Published November 25, 2010

Citation: Jain V, Vyas AS (2010) Cervical Neoplasia-Cyto-Histological Correlation (Bethesda System) A Study of 276 Cases. J Cytol Histol 1:106. doi:10.4172/2157 7099.1000106

Copyright: (c) 2010 Jain V, et al. This is an open-access article distributed unde the terms of the Creative Commons Attribution License, which permits unrestricted use, distribution, and reproduction in any medium, provided the original author and source are credited. 
Citation: Jain V, Vyas AS (2010) Cervical Neoplasia-Cyto-Histological Correlation (Bethesda System) A Study of 276 Cases. J Cytol Histol 1:106. doi:10.4172/2157-7099.1000106

Page 2 of 3

smears were made by scrapping the cervix from the squamo-columnar junction with the help of cotton swab stick. The endocervical smears were made by rotating the swab stick in clockwise direction in squamo-columnar junction.

The smears were immediately fixed in ether: ethyl alcohol fixative for 30-45 minutes and sent to cytology lab for further processing, where the slides were stained with Papanicolaou stain. Corresponding histology slides were stained with H \&E stain.

\section{Statistical analysis used}

The total number of smears throughout two years was 4805 . Out of these, 4251 smears were inflammatory, 287 were unsatisfactory, and the remaining 276 smears showed epithelial cell lesions. The cytology findings and corresponding histology findings were studied. Histology was taken as the gold standard. The PAP smears were classified according to The Bethesda 2001 system and the histological findings were classified according to The WHO classification 2003. The concordance rate for each variable was calculated.

\section{Discussion}

A total of 276 cases were studied. Retrospectively examination of histology sections and corresponding cytology slides (PAP smears) was done. The concordance rate for each lesion was calculated according to the Bethesda 2001 system. The majority cases were of squamous cell carcinoma on both cytology and histology (69.5\%). The overall concordance rate of the study was $70.7 \%$. The discordant cases being 81 i.e. $29.3 \%$. The sensitivity was $78 \%$, specificity $26.9 \%$, positive predictive value $91.1 \%$, negative predictive value $11.3 \%$ and accuracy $73.2 \%$. The concordance rate for squamous cell carcinoma was $83.6 \%$ with sensitivity $84 \%$. The specificity was high i.e. $90.4 \%$ and positive predictive value $95.3 \%$. The concordance rate for cervical intraepithelial neoplasia was less i.e. $42.2 \%$ with sensitivity
$42 \%$, specificity $36.8 \%$ and positive predictive value $61.3 \%$. For adenocarcinoma the concordance rate was $50 \%$. For other lesions like adenosquamous carcinoma, squamous papilloma and malignant mixed mullerian tumor, the discordance rate was $100 \%$. For radiation induced changes the concordance rate was $66.7 \%$, cervicitis $20 \%$ and normal histology $60 \%$.

In the present study, the overall concordance rate was $70.7 \%$ which is comparable to other studies e.g. Study by Nawaz (a study from Aga Khan University, Pakistan) where the concordance rate was $74 \%$. The other studies like Saha's, Yeoh \& Rasbridge has concordance rates as $60 \%, 52 \%$ and $81.2 \%$ respectively (Table 3 ).

From the Table 4 , it is seen that concordance rate for squamous cell carcinoma in our study is $83.6 \%$ as compared to the studies by Saha, Yeoh and Nawaz in which the rate is $100 \%, 60 \% \& 97.33 \%$ respectively.

Out of the 29 discordant cases, 14 cases were underdiagnosed on cytology as HSIL, 9 cases were called as negative/benign and ASCUS, ASC-H \& LSIL were the diagnoses in 2 cases each. The main factor for underreporting was less cellularity with hemorrhagic background. Repeat smears from these patients showed frank malignancy in 6 cases and they were later on upgraded as squamous cell carcinoma. Rest of the 23 smears showed air drying and fixation artifacts and so the diagnosis of LSIL or less persisted.

In Saha's study [8] the overall concordance rate was $60 \%$ and that for squamous cell carcinoma were $100 \%$.

In Yeoh's study [7], 83 matching cases were between cytological and histological records. The overall concordance rate was $52 \%$ and that for squamous cell carcinoma was $60 \%$. Out of 18 discordant cases, 8 were over reported and 10 underreported. A major component of false negative rate appeared to be sampling and preparation artifacts.

\begin{tabular}{|l|l|l|l|l|}
\hline Name of study & Audit by Simon Rasbridge ${ }^{6} 1995$ & Yeoh study 1997 & Saha's study ${ }^{8} 2005$ & Nawaz study 2005 \\
\hline Concordance rate & $81.2 \%$ & $52 \%$ & $60 \%$ & $74 \%$ \\
\hline
\end{tabular}

Table 3: Comparison of overall concordance rate with other studies

\begin{tabular}{|c|c|c|c|c|c|}
\hline & Saha's study ${ }^{8} 2005$ & Yeoh study ${ }^{7} 1997$ & Nawaz study ${ }^{9} 2005$ & Audit by Simon Rasbridge 61995 & Present study \\
\hline ASCUS & 0 & 0 & 0 & 0 & $3(1.02 \%)$ \\
\hline $\mathrm{ASCH}$ & 0 & 0 & 0 & 0 & $4(2.06 \%)$ \\
\hline Negative /benign & 0 & $1(9.09 \%)$ & 0 & 5 & $9(4.61 \%)$ \\
\hline LSIL & 0 & $1(9.09 \%)$ & 0 & 6 & $2(1.02 \%)$ \\
\hline HSIL & 0 & $3(27.2 \%)$ & $2(2.7 \%)$ & 10 & $14(7.17 \%)$ \\
\hline Squamous cell carcinoma & $3(100 \%)$ & $6(54.5 \%)$ & $73(97.3 \%)$ & $123(91.8 \%)$ & $163(83.6 \%)$ \\
\hline Total cases & 3 & 11 & 75 & 134 & 195 \\
\hline
\end{tabular}

Table 4: Comparison of the concordance for squamous cell carcinoma in various studies.

\begin{tabular}{|c|c|c|c|c|c|}
\hline \multicolumn{6}{|c|}{ CIN II /III } \\
\hline & Yeoh $^{7} 1997$ & Sodhani ${ }^{10} 2004$ & Saha $^{8} 2005$ & Nawaz $^{9} 2005$ & Present study \\
\hline Negative/benign & $10(7.81 \%)$ & 0 & $28(27.45 \%)$ & $3(7.5 \%)$ & $4(8.69 \%)$ \\
\hline ASCUS & $10(11.11 \%)$ & $4(4 \%)$ & 0 & 0 & $9(22.58 \%)$ \\
\hline ASC-H & 0 & $6(6 \%)$ & 0 & 0 & $1(3.22 \%)$ \\
\hline LSIL & $32(11.11 \%)$ & $16(16 \%)$ & 19(18.63\%) & $10(25 \%)$ & $10(32.2 \%)$ \\
\hline HSIL & $72(55.56 \%)$ & $74(74 \%)$ & $72(70.59 \%)$ & $22(55 \%)$ & $6(26.08 \%)$ \\
\hline SCC & $4(3.125 \%)$ & 0 & $3(2.95 \%)$ & $5(12.50 \%)$ & $1(65.21 \%)$ \\
\hline Total & 128 & 100 & 102 & 40 & 31 \\
\hline
\end{tabular}

Table 5: Comparison of concordance rate for cervical intraepithelial neoplasia cin ii \& iii with other studies.

\begin{tabular}{|l|l|l|l|l|}
\hline CIN I & Yeoh 71997 & Saha $^{8} 2005$ & Nawaz \\
\hline & 11 & 0 & 0 & \\
\hline ASCUS & $19(4.11 \%)$ & $2(25 \%)$ & $18(32.2 \%)$ \\
\hline Negative/benign & $37(50.7 \%)$ & $6(75 \%)$ & $28(50 \%)$ \\
\hline LSIL & $19(43.9 \%)$ & 0 & $8(14.3 \%)$ & $3(21.4 \%)$ \\
\hline HSIL & 0 & 0 & $2(3.57 \%)$ \\
\hline SCC & 86 & 8 & 56 & $9(64.3 \%)$ \\
\hline Total & & 8 & 0 & 0 \\
\hline
\end{tabular}

Table 6: Comparison of concordance rate for cervical intraepithelial neoplasia cin I with other studies. 
Citation: Jain V, Vyas AS (2010) Cervical Neoplasia-Cyto-Histological Correlation (Bethesda System) A Study of 276 Cases. J Cytol Histol 1:106. doi:10.4172/2157-7099.1000106

21 biopsies from 22 cases reported as normal on PAP showed CIN. So the conclusion drawn was to decrease false negative rate, smears should be repeated at regular intervals. Error rate is negligible with three normal consecutive annual smears. It is appropriate to take the test with higher degree of abnormality as the correct result.

The study by Nawaz $[7,9]$ showed overall concordance rate of $74 \%$ and $97 \%$ concordance for squamous cell carcinoma. A total 8 numbers of cases were discrepant. The causes for discrepancies were mainly sampling error, air drying and blood or inflammation obscuring the cellularity.

The Table 5 shows that out of 31 cases of cervical intraepithelial neoplasia II \& III, 16 cases were concordant on cytology. So the concordance rate is $32.3 \%$. The remaining 25 cases were discordant out of which 24 cases were underdiagnosed on cytology and one case overdiagnosed the main cause for discrepancy being inadequacy of smear and drying artifact.

In the study by Yeoh [7], total of 128 cases were diagnosed on histology as CIN II/III out of which 72 cases were concordant. Out of the remaining 56 cases, 52 were underdiagnosed on cytology and 4 cases were over -diagnosed as squamous cell carcinoma. The concordance rate was $74.6 \%$.

The concordance rates for Nawaz [9], Gupta and Sodhani [10] study were $92 \%$ and $74 \%$ respectively.

In the study by Gupta and Sodhani [10] titled as "Why is high grade squamous intraepithelial neoplasia underdiagnosed on cytology in a quarter of cases? analysis of smear characteristics in discrepant cases cervical smears of 100 histology proven cases of cervical intraepithelial neoplasia III (CIN III) were retrieved and reviewed to study Cytohistological agreement in high grade lesions. Cytology was able to correctly identify 74 HSILs whereas in 26 cases a diagnosis of LSIL or below was given on review, 16 of these cases were reclassified as HSIL on cytology while 10 cases showed persistent diagnosis of LSIL. 12/16 (75\%) cases represented interpretative errors. Sampling error was 7/10 and air drying 5/10 were found in underdiagnosed cases.

From Table 6 the concordance rate for CIN I in present study is $64.3 \%$ as compared to Saha's study having the rate of $75 \%$. In Yeoh study, it is $50.68 \%$ and the study by Nawaz gives the concordance rate of $50 \%$.

The positive predictive value for CIN in present study was $61.3 \%$ as compared to the study by Nawaz having the rate of $76 \%$.

\section{Results}

In the present study the overall sensitivity is $78 \%$, specificity
$26.9 \%$, positive predictive value $91.1 \%$ and accuracy of $73.2 \%$.

$$
\begin{aligned}
& \text { Sensitivity }=\frac{A}{A+C}=\frac{195 \times 100}{195+55}=78 \% \\
& \text { Specificity }=\frac{D \times 100}{D+B}=\frac{7 \times 100}{7+19}=26.9 \% \\
& \text { Positive Predictive Value }=\frac{A \times 100}{A+B}=91.1 \% \\
& \text { Accuracy }=\frac{A+D \times 100}{A+B+C+D}=73.2 \% \\
& \text { Negative Predictive Value }=\frac{D \times 100}{C+D}=11.3 \%
\end{aligned}
$$

The overall concordance of the study was $70.7 \%$. The discordant cases were $81(29.3 \%)$. The sensitivity was $78 \%$ \& positive predictive value $91.1 \%$

\section{Conclusion}

The study provides a clue to assess the internal quality of cytology reporting. The PAP smear has good sensitivity and specificity and positive predictive value in detecting high grade lesions and malignancy. This is particularly effective in our set-up as majority of the patients present with grade III and IV lesion. The sensitivity for cervical intraepithelial neoplasia is low but it can be increased by adequate sampling and avoiding technical errors like air drying and fixation artifacts. The discrepancy can be minimized by following the Bethesda system for adequacy of sampling.

\section{References}

1. Papanicolaou GN (1928) New cancer diagnosis proc $3^{\text {rd }}$ Race Betterment conference Battle Greek, Michigan, 528-530.

2. Dehner LP (1993) Cervicovaginal cytology, false negative result and standard of practice. Am J Clin Pathol 99: 45-47.

3. National cancer institute workshop report (1989) The 1988 Bethesda system for reporting cervical/vaginal cytological diagnosis. JAMA 262: 931-934.

4. Broder S (1992) From the National Institutes of Health. JAMA 267: 1892.

5. Crothers BA (2005) The Bethesda System 2001: update on terminology and application. Clin Obstet Gynecol 48: 98-107.

6. One year audit by Simon (1995) Rasbridge Acta cytologica 39: 648-651.

7. Yeoh GPS, Chan KW (1997) The accuracy of Papanicolaou smear prediction: Cytohistological correlation of 283 cases. HKMJ 3: 373-376.

8. Saha R, Thapa M (2005) Correlation of cervical cytology with cervical histology Kathmandu univ Med J (KUMJ) 3: 222-224.

9. Nawaz FH, Aziz AB, Perwez S, Rizvi JH (2005) Prevalence of abnorma papanicolaou smears and cytohistological correlation. A study from Aga Khan University hospital, Pakistan. Asia-Pacific Journal of clinical oncology 1: 128132.

10. Gupta S, Sodhani P (2004) Why is high grade squamous intraepithelia neoplasia under-diagnosed on cytology in a quarter of cases? Analysis of discrepant cases' Indian J Cancer 41: 104-108. 